\title{
Measurement of infection efficiency of a major wheat pathogen using time-resolved imaging of disease progress
}

Article

Accepted Version

Karisto, P., Dora, S. and Mikaberidze, A. (2019) Measurement of infection efficiency of a major wheat pathogen using timeresolved imaging of disease progress. Plant Pathology, 68 (1). pp. 163-172. ISSN 0032-0862 doi:

https://doi.org/10.1111/ppa.12932 Available at https://centaur.reading.ac.uk/86103/

It is advisable to refer to the publisher's version if you intend to cite from the work. See Guidance on citing.

To link to this article DOI: http://dx.doi.org/10.1111/ppa.12932

Publisher: Wiley-Blackwell

All outputs in CentAUR are protected by Intellectual Property Rights law, including copyright law. Copyright and IPR is retained by the creators or other copyright holders. Terms and conditions for use of this material are defined in the End User Agreement.

www.reading.ac.uk/centaur 
Central Archive at the University of Reading

Reading's research outputs online 


\title{
Measurement of infection efficiency of a major wheat \\ 5 pathogen using time-resolved imaging of disease progress
}

\author{
P. Karisto*十, S. Dora† and A. Mikaberidze
}

10 Plant Pathology Group, Institute of Integrative Biology, ETH Zurich, Zurich, Switzerland.

*Corresponding author: P. Karisto, email: petteri.karisto@usys.ethz.ch †Contributed equally

Running head: Zymoseptoria infection efficiency

Keywords: epidemiology, quantitative resistance, disease management, crop protection, host-pathogen interaction, wheat diseases 


\section{Abstract}

Infection efficiency is a key epidemiological parameter that determines the proportion of pathogen spores able to infect and cause lesions once they have landed on a susceptible plant tissue. In this study, we present a new method to measure infection efficiency of Zymoseptoria tritici using a

25 replicated greenhouse experiment. $Z$. tritici is a fungal pathogen that infects wheat leaves and causes Septoria tritici blotch (STB), a major disease of wheat worldwide.

We devised an original experimental setup, where we (i) attached living wheat leaves to metal plates allowing for time-resolved imaging of

30 disease progress in planta. Since lesions were continuously appearing, expanding and merging during the period of up to three weeks, daily measurements were necessary for accurate counting of lesions. We also (ii) used reference membranes to characterize the density and the spatial distribution of inoculated spores on leaf surfaces. In this way, we captured

35 the relationship between the number of lesions and the number of viable spores deposited on the leaves and estimated the infection efficiency of about $4 \%$ from the slope of this relationship.

Our study provides a proof of principle for an accurate and reliable measurement of infection efficiency of $Z$. tritici. The method opens

40 opportunities for determining the genetic basis of the component of quantitative resistance that suppresses infection efficiency. This knowledge would improve breeding for quantitative resistance against STB, a control measure considered more durable than deployment of major resistance genes. 


\section{Introduction}

Foliar fungal pathogens of plants produce immense numbers of spores (Sache $\&$ de Vallavieille-Pope, 1995), but only a small fraction of them causes new infections. Most of the spores do not land on plant tissue that

50 they can infect and are "lost" in the environment. Even among the spores that succeed in finding suitable host tissue, only a moderate proportion will infect and cause lesions. This proportion is determined by the infection efficiency, defined as the probability of an individual spore that lands on a susceptible host tissue to cause a lesion. Infection efficiency is

55 one of the key determinants of the pathogen's rate of transmission. From the perspective of the host, the host's ability to suppress the infection efficiency is a major component of quantitative resistance that reduces the rate of epidemic development (Parlevliet, 1979).

In this study, we present a novel method to measure infection efficiency

60 of Zymoseptoria tritici (formerly known as Mycosphaerella graminicola). This fungal pathogen causes Septoria tritici blotch (STB), a major disease of wheat (Triticum aestivum) in Europe (Jørgensen et al., 2014) and worldwide (Orton et al., 2011). Even controlled epidemics of the disease can lead to notable yield losses if the environmental conditions favour the

65 development of the disease (Fones \& Gurr, 2015). STB is controlled mainly by foliar fungicide applications and deployment of disease resistant wheat varieties. However, acquired fungicide resistances are spreading in the genetically diverse population of $Z$. tritici and diminishing the efficacy of fungicide treatments (Fraaije et al., 2005; Zhan et al., 2006). Similarly, the

70 pathogen has the capacity to adapt to both major gene resistance (Cowger et al., 2000) and quantitative resistance (Cowger \& Mundt, 2002). Quantitative resistance is generally thought to be more durable than major gene resistance (St.Clair, 2010). Accurate phenotypic 
characterization of infection efficiency has the potential to improve

75 breeding for quantitative resistance against STB.

The infection cycle of $Z$. tritici starts with the deposition of inoculum composed of wind-dispersed ascospores and rain-splash dispersed pycnidiospores on the leaves (Suffert et al., 2011). On the leaf surface, germinating spores penetrate the plant tissue through stomata, enter the 80 apoplast and start to colonise the leaf in an asymptomatic manner. During a latent period of roughly two weeks (Kema et al., 1996), the fungus evades recognition by the plant defence mechanisms. Once the fungus has colonised the plant tissue, it starts to produce necrotic lesions within which it produces pycnidia and eventually releases new pycnidiospores

85 that disperse further and generate secondary infections (Orton et al., 2011).

Infection efficiency can be quantified as the ratio between the number of lesions visible on a leaf and the number of viable spores deposited on a leaf surface through artificial inoculation. Therefore, to measure infection

90 efficiency one needs to measure accurately the number of viable spores deposited on the leaf and the resulting number of lesions formed on the leaf. Measurements of infection efficiency were conducted in many airborne fungal pathogens including several rust species. Sache $\&$ de Vallavieille-Pope (1995) discuss data on 22 species. Methods used to

95 estimate the number of deposited spores include counting spores directly on the leaf surface (Mehta \& Zadoks, 1970; Melching et al., 1988), weighing the inoculum (Sache, 1997), and using a known volume of liquid inoculum in which the concentration of spores was measured (Levy, 1989; Shine \& Jarriel, 1990).

100 To achieve a precise measurement of infection efficiency, not only the amount of inoculum but also its spatial distribution across the leaf surface needs to be characterized. When fungal spores arrive to the surface of the 
leaf in groups, for example as droplets containing several spores, an infection event cannot be with certainty attributed to a single spore. The

105 same argument holds in the case of high densities of spores on the leaf surface when many spores are likely to be present within the area covered by a typical lesion. In these cases, the infection efficiency cannot be determined by simply dividing the number of lesions by the number of deposited viable spores. Moreover, possible interaction between spores

110 when producing lesions may bias the estimate of infection efficiency. Thus, a spatially uniform, precisely measured and sufficiently low density of spores is needed for a reliable measurement. Different approaches to achieve suitable distribution of inoculum include spore settling towers (Brown \& Kochman, 1973), atomizers (Statler \& McVey, 1987) and

115 paintbrushes (Melching et al., 1988).

One of the challenges in estimating the infection efficiency of $Z$. tritici is that spores of this pathogen are not well visible on wheat leaves and cannot be counted directly on the leaf surface. The only measurement of infection efficiency of $Z$. tritici available to date was reported by Fones et

120 al. (2015). They applied spore suspensions to leaves and spread the inoculum across the leaf manually with a finger covered by a rubber glove. The estimate of the infection efficiency was then given by the ratio between the number of individual lesions observed on the leaves and the total number of spores contained in the inoculated suspension. However,

125 Fones et al. (2015) characterized neither the spatial distribution of the deposited spores across the leaf surface nor the viability of spores. In addition, the number of lesions was only measured at a single time point, which may have led to an underestimation of lesion numbers, if not all lesions appeared or if some lesions already merged by this time.

130 In this study, we developed a method to measure the infection efficiency of $Z$. tritici accurately, with a relatively low effort and low cost. One of the key aspects of the method was the use of reference membranes that 
provided information on the density and the spatial distribution of viable spores deposited on leaves. In our setup, the leaves were attached to

135 metal plates, so that the inoculated area of the leaves was easy to observe and image during the infection. This allowed us to record the appearance of individual lesions with temporal resolution from the time when first lesions started to appear, which improved crucially the accuracy in counting lesions.

\section{Materials and Methods}

\section{Plant and fungal material}

We planted winter wheat (Triticum aestivum) cultivar Drifter seeds in $6 \times 6 \times 11 \mathrm{~cm}$ pots containing soil substrate (Jiffy soil substrate GO PP7, Netherlands) and watered them regularly. Cultivar Drifter was classified as

145 susceptible to STB according to multi-year field trials in Germany (Risser, 2010) and also was found to be one of the most susceptible among 335 elite European wheat cultivars exposed to the diverse natural $Z$. tritici population in a recent field experiment in Switzerland (Karisto et al., 2018). The plants were fertilized ten days after sowing with 1 I of fertilizer

150 solution (Wuxal Universal-Dünger, Maag-Garden, Switzerland; $1 \mathrm{ml} / \mathrm{l}$ diluted in tap water) per 20-24 pots. The plants were grown in the greenhouse with the light/dark cycle of $16 / 8$ hours, the relative humidity of $70 \%$ and the temperature of $18 / 15^{\circ} \mathrm{C}$. After inoculation, the plants were trimmed twice a week by cutting the newly emerged leaves above

155 the inoculated second leaf.

To prepare fungal spores for the inoculation of the plants, $Z$. tritici strain ST_CH3_99_3D7 (short identifier 3D7; Zhan et al., 2002; Septoria tritici blotch network, 2017) blastospores were grown in $50 \mathrm{ml}$ yeast-sucrosebroth (10 g/l sucrose, $10 \mathrm{~g} / \mathrm{l}$ yeast extract, $50 \mathrm{mg} / \mathrm{l}$ kanamycin) for 5 days

160 at $18^{\circ} \mathrm{C}$ in the dark. We chose this strain because it is known to be highly 
virulent on cultivar Drifter under greenhouse conditions (for example Palma-Guerrero et al., 2016) and is well characterized both genetically and phenotypically. The liquid culture was then filtered, pelleted and suspended in sterile water and the concentration of spores was

165 determined using a KOVA Glasstic Slide counting chamber. The inoculum was diluted to achieve the required concentrations and $1 \mathrm{ml} / \mathrm{l}$ of Tween 20 (Biochemica, Applichem Gmbh, Germany) was added. The spore suspension was kept on ice until the inoculation of the plants was conducted on the same day.

\section{Experimental procedures}

The whole experiment was repeated twice as described below. Each repetition can be considered as an independent biological replicate. For clarity, we refer to the two biological replicates as the "first experiment" and the "second experiment".

175 We inoculated second leaves of sixteen days old wheat seedlings. For this purpose, the pots were placed into a tray with an aluminium plate in the middle as shown in Fig. 1. The leaves to be inoculated were arranged in four sets containing 5-8 leaves each on an aluminium plate and attached with eight elastic threads. Reference membranes (Whatman ${ }^{\mathrm{TM}} 3 \mathrm{MM} \mathrm{Chr}$

180 Chromatography paper, Fisher scientific) were attached to plates next to each leaf set. Spore suspension was applied while moving the paint gun sprayer (RevolutionAir, Fini Nuair, Italy) twice along the plate. The sprayer was operated with 20 psi pressure (adjusted with the pressure reducer Filterdruckminderer R 1/4", Einhell, Germany) and the minimal flow rate to

185 maximize the atomization of the spore suspension and the uniformity of coverage.

The plants were infected with spore suspensions that contained $5 \times 10^{2}$, $10^{3}, 5 \times 10^{3}, 10^{4}, 5 \times 10^{4}$, and $10^{5}$ spores $/ \mathrm{ml}$. Different concentrations of spores in the suspension corresponded to different treatments. A control 
190 treatment contained no spores. Each treatment was applied to a single tray of plants that contained four leaf sets with reference membranes placed on both sides of each leaf set. The number of leaves per treatment ranged from 22 to 30 . After the inoculation, the plants were enclosed in plastic bags for three days to keep them at a high humidity. Plants were

195 maintained in the greenhouse until the time when observation of new lesions was no longer possible due to coalescence of lesions or the onset of natural senescence.

After the inoculation, reference membranes were transferred from infection plates to yeast-malt-agar ( $12 \mathrm{~g} / \mathrm{l}$ bacteriological agar, $4 \mathrm{~g} / \mathrm{l}$ yeast

200 extract, $4 \mathrm{~g} / \mathrm{l}$ malt extract, $4 \mathrm{~g} / \mathrm{l}$ sucrose, $50 \mathrm{mg} / \mathrm{l}$ kanamycin) and incubated for 5- 6 days in darkness at $18^{\circ} \mathrm{C}$. After the incubation, we measured the density of $Z$. tritici colonies by counting them within two or three squares of $1 \mathrm{~cm}^{2}$ on digital images of each reference membrane (Appendix S1, Fig. S1 shows examples of colony images). In the second

205 experiment, in treatment with $10^{5}$ spores $/ \mathrm{ml}$ we used $0.25 \mathrm{~cm}^{2}$ squares. To determine the nature of units that formed fungal colonies, we inspected them under the microscope. When we sprayed the same spore suspension on a microscopy slide during the inoculation, we observed only individual spores without any clumps (we observed 82 spores in total,

210 Appendix S1, Fig. S2). This indicated that each colony grew from an individual spore and therefore individual spores acted as colony forming units (CFUs). At the same time, colony densities did not differ significantly between reference membranes that belonged to the same treatment indicating that the density of spores was the same on reference

215 membranes and on the leaves in their vicinity. We used the colony density measured in this way as an estimate of the density of viable spores in the inoculated areas of leaves assuming that each viable spore was able to form a colony on rich medium under favourable conditions. We provide more details on the inoculation method in Appendix S1. 
To estimate the number of CFUs that landed on each leaf, the areas of leaves were measured. Photographs of leaf sets were taken on the day of inoculation and leaf areas were then measured using Adobe Photoshop CS6. Numbers of CFUs on each leaf were calculated by multiplying the 225 mean densities of colonies on reference membranes on each side of the leaf set by the areas of individual leaves.

To observe the development of lesions, we inspected the infected leaves every day. After the onset of lesion appearance, digital images of leaf sets were captured every day until majority of the leaves became covered by

230 lesions or naturally senescent. Lesions were counted manually on digital images. Newly appeared lesions were marked in the images on the day of their first appearance and the markers were transferred to images captured on the following days in order to avoid double counting of lesions. The total number of lesions on each leaf was calculated from the

235 data on daily appearance of lesions. The lesion density (number of lesions per $\mathrm{cm}^{2}$ of leaf area) in each treatment was calculated for each leaf separately based on the total number of lesions that appeared until the last day of observation [34 days post inoculation (DPI) for the first experiment and 36 DPI for the second experiment]. Several leaves were

240 damaged during the experiment and removed.

\section{Data analysis}

Analysis of the data was conducted in R (R Core Team, 2017). We evaluated differences within treatments and between treatments in terms of lesion density and spore density (density of viable spores on the leaf

245 surface). For this purpose, we used two separate analyses of variance (ANOVA). To estimate the infection efficiency of spores that were applied to the leaves, we performed linear regression for the dependency between the spore density and the lesion density on the leaf surface. At 
high concentrations, we expected to observe a saturation of this

250 dependency because there is a limit to the number of lesions that can form on a single leaf due to finite space and/or resources. For this reason, we used the Akaike information criterion (AIC) to compare a linear model that does not include saturation to a nonlinear model that accounts for possible saturation. AIC balances the goodness of fit with the number of

255 parameters (or complexity) of the model. In this way, we determined whether saturation occurred at high spore densities and identified the range of spore densities that is not affected by saturation. As the nonlinear model, we used the classical Michaelis-Menten model, $y=a x /(1+b x)$, where $y$ is the lesion density, $x$ is the spore density, and a

260 and $b$ are the model parameters. As the linear model, we used the function $y=a x$. We fitted the two models to the data of each experiment with the nonlinear least squares minimization method (routine nls in R). The data points at high densities that were in the range of saturation according to the AIC score were excluded and the infection efficiency was

265 estimated as the slope of the best-fitting linear function.

To compare the infection efficiency between the two experiments, we tested whether the two slopes were significantly different. First, we used the analysis of covariance (ANCOVA) to test whether there was a significant interaction between the slope and the experiment. Second, we

270 tested whether the two slopes were different using the t-test for the slope difference (Zar, 2010).

\section{Results}

\section{Experimental design}

275 The spore suspension appeared to be uniformly distributed across the surfaces of leaves and reference membranes when spraying with the 
paint gun sprayer. The uniformity of the inoculum was quantified by measuring the spatial distribution of fungal colonies on references membranes. The colony densities on reference membranes that belonged

280 to the same treatment exhibited a slight variation but did not differ significantly (ANOVA, p-values between 0.087 and 0.92). As expected, the colony density (averaged over the reference membranes belonging to the same treatment) mostly increased monotonically with increasing the concentration of spores in the suspension (inoculum concentration), as

285 can be seen from Table 1. Only the second lowest spore concentration in the second experiment exhibited a non-monotonic pattern. The spraying resulted on average in $1-2$ colonies $/ \mathrm{cm}^{2}$ on the reference membranes per 1000 spores/ml in the spore suspension (Appendix S1, Fig. S3).

The elastic threads kept the leaves sufficiently flat and stable during the

290 progress of the disease, which enabled accurate observation of the inoculated area of leaves over time.

\section{Appearance of lesions}

According to our observations, lesions were continuously appearing on leaves during the time span of about three weeks (Fig. 2). Figures $2 \mathrm{a}$ and

$2952 \mathrm{~b}$ show the rate of appearance of new lesions over time, while Figures $2 \mathrm{c}$ and $2 \mathrm{~d}$ show the change over time in the total number of lesions. In every treatment, the dynamics was qualitatively similar. First, lesions started to appear at a slow rate, then the rate of their appearance increased, reached its maximum and eventually dropped to zero (Fig. 2a, 2b). After

300 this time, no new lesions appeared, hence the total number of lesions remained constant (Fig. 2c, 2d).

At higher spore densities, lesions started to appear earlier compared to lower spore densities [compare for example purple curves (squares) to cyan curves (stars) in Fig. 2; the difference was not tested statistically].

305 Lesions that appeared earlier started to grow and merge with lesions that 
appeared in their vicinity a few days later (Fig. 1). Without daily observations, we would not be able to distinguish them. Leaves inoculated with higher concentrations of spores carried larger final numbers of lesions. For this reason, in treatments with highest spore densities, lesions

310 covered the entire leaf surface soon after their appearance and we could not observe any further appearance of lesions (Fig. 2). On the contrary, in treatments with lower spore densities, numbers of lesions were smaller and we could observe individual lesions over a longer time.

\section{Measurement of infection efficiency}

315 To determine the infection efficiency, we characterized the numbers of viable spores applied to the leaves and measured the numbers of lesions that subsequently appeared on the leaves (Table 1 ). In different treatments, the mean spore density (the number of spores per unit leaf area) ranged from 1.3 to 117 spores $/ \mathrm{cm}^{2}$ in the first experiment and from

3203.4 to 223 spores $/ \mathrm{cm}^{2}$ in the second experiment. The mean lesion density (the number of lesions per unit leaf area) measured on the final day of observation also varied between treatments. This quantity ranged from 0.092 to 2.8 lesions $/ \mathrm{cm}^{2}$ in the first experiment (measured at $34 \mathrm{DPI}$ ) and from 0.066 to 5.2 lesions $/ \mathrm{cm}^{2}$ in the second experiment (measured at 36

325 DPI).

Comparison between linear and nonlinear models based on AIC favoured the nonlinear dependency of the lesion density on the spore density. The AIC scores were 223 and 108 in the first experiment, 446 and 300 in the second experiment for the linear and the nonlinear models, respectively.

330 This means that the dependency exhibits a substantial saturation (dashed lines in Fig. 3a). To determine the range of spore densities that is not affected by the saturation, we excluded the treatment with the highest spore densities (117-119 spores/ $\mathrm{cm}^{2}$ in the first experiment and 212$242 \mathrm{spores} / \mathrm{cm}^{2}$ in the second experiment). Now, the comparison favoured 335 the linear model (AIC score -31.1 and -29.8 in the first experiment; 157 
and 159 in the second experiment for linear and nonlinear models, respectively). This indicates that saturation only played a role at the highest spore densities, while at lower spore densities the lesion density depended linearly on the spore density and was not affected by

340 saturation. We used this range (all data except for the treatment with $10^{5}$ spores/ml that corresponds to highest spore densities) to determine the infection efficiency.

We estimated the infection efficiency as the slope of the linear part of the relationship between the lesion density and the spore density (solid lines

345 in Fig. 3$)$. This yielded the estimates $(3.8 \pm 0.1) \%$ in the first experiment $\left(R^{2}=0.95\right)$ and $(4.3 \pm 0.2) \%$ in the second experiment $\left(R^{2}=0.95\right)$, where the uncertainties represent $95 \%$ confidence intervals. According to ANCOVA, the slopes differed between the two experiments $\left(p=1.1 \times 10^{-6}\right.$, $\mathrm{F}=24.7, \mathrm{df}=1$ ) and the t-test (Zar, 2010) showed the same result ( $p=$

$3501.8 \times 10^{-4}, \mathrm{t}=4.6, \mathrm{df}=327$ ). The full dataset can be accessed from the Dryad Digital Repository xxx.

\section{Discussion}

We presented an accurate and reliable method for measuring the infection efficiency of $Z$. tritici. Main advantages of the method include the

355 use of reference membranes to estimate densities of viable spores deposited on leaves and the daily imaging of leaves attached to metal plates. Counting colonies on reference membranes allowed us to estimate accurately densities of inoculated viable spores and confirmed the uniformity of their spatial distribution on leaf surfaces. Attaching leaves to

360 metal plates allowed for efficient daily observations that resulted in accurate counting of individual lesions. Stability of the leaves ensured that the inoculum was not moving across the leaf surface and helped to observe the appearance of lesions from daily photographs. The method is 
based on commonly used low-cost equipment, which makes it affordable 365 and easy to modify.

Each of the individual components of our method has been used in the literature previously. Similar ideas on estimating densities of viable spores with reference plates were used by Chakraborty et al. (1990) in a different pathosystem; a range of inoculum concentrations, including low

370 concentrations, were used by Fones et al. (2015) in this pathosystem; attached leaf assays were previously used in this pathosystem for example by Keon et al. (2007) and Lee et al. (2014); time-resolved imaging was used before in fungal biology for example in studies of spore ejection of basidiomycete fungi (Noblin et al., 2009). In addition,

375 numerous studies measured the ability of fungal spores to grow colonies under conducive conditions in vitro to estimate the viability of spores: for example Valsecchi et al. (2017) in Aspergillus fumigatus and King et al. (2017) in Z. tritici. Unlike the previous studies in this pathosystem that used cotton swabs to deploy the inoculum suspension on leaves (Lee et

380 al., 2014) or pipetted a well-defined volume of spore suspension onto the leaf and spread it using a gloved finger (Fones et al., 2015), we used a paint gun sprayer [comparable to Statler \& McVey (1987) for example] that produced an evenly distributed "fog" of tiny droplets. This allowed us to characterize the density of viable spores and to verify the uniformity of

385 their spatial distribution using reference membranes. Thus, the novelty of our work lies not so much in each individual component of the method per se, but rather in a specific way we combined these methodological aspects that allowed us to obtain accurate estimates of the pathogen's infection efficiency.

390 In contrast to some of the earlier measurements of infection efficiency in plant pathogens that quantified the total number of spores in the applied inoculum, we estimated the number of viable spores in the applied inoculum. This is because when estimating the infection efficiency, we 
need to quantify the proportion of viable spores that have caused lesions.

395 We estimated the density of viable spores from the density of fungal colonies on agar plates (as described in Materials and Methods).

Depositing spores of fungal pathogens on solid medium, incubating them under optimal conditions and counting the number of resulting colonies is an established method to evaluate the viability of fungal spores that was

400 previously used in Z. tritici (King et al., 2017) and in other pathosystems, for example in Aspergillus fumigatus, (Valsecchi et al., 2017). Viability of spores is likely to be higher on agar than on the leaf surface because (i) agar plates contain more nutrients and (ii) plates are maintained under constant environmental conditions that are close to optimal for the fungus

405 in contrast to the leaf surface in the greenhouse that is exposed to various stress factors such as variable humidity, temperature and light with a wide frequency spectrum including UV. This difference is acceptable because our goal was to estimate the maximum number of viable spores in the sprayed suspension. Decreased survival of spores on leaves

410 compared to agar is included in the estimate of the infection efficiency. At the same time, there could be factors that promote germination and thus the observed viability of the spores on the leaf surface. However, to our knowledge, there is no evidence for host-specific cues or other factors promoting germination of $Z$. tritici spores on the leaves. For this reason,

415 we assumed that the maximum viability of spores was captured by the method described.

We found that the relationship between the density of fungal colonies on reference membranes and the concentration of spores in the suspension was monotonic: colony density increased at higher concentrations of

420 spores. An exception to this tendency was observed only for the second lowest spore concentration in the second experiment, which exhibited a non-monotonic pattern (see Table 1 ). This non-monotonic pattern could result from a non-homogeneous distribution of spores in the suspension during the spray, if the spores had time to settle down inside the sprayer 
425 tank. At low concentrations of spores, the dependency may also be sensitive to slight variations in the manual movement of the sprayer between treatments. Noticeable deviations from a perfect linear fit between the spore concentration in the suspension and the density of colonies on plates were observed for example in treatment $10^{4} \mathrm{spores} / \mathrm{ml}$,

430 which resulted in higher colony density than expected (Appendix S1, Fig. $\mathrm{S} 1)$. All these observations indicate that the measurement of the concentration of spores in the inoculum suspension is not sufficient to determine reliably the actual density of spores on leaf surfaces. Therefore, an important aspect of the method is to characterize the density of viable

435 spores present on the leaves after every spray of the inoculum suspension. We achieved this conveniently by counting colonies on reference membranes.

Our measurements show that lesions did not all appear within a narrow time span of a few days. On the contrary, they were continuously

440 appearing during a period of up to three weeks (see Fig. 2), even though we used a single pathogen strain to infect a single host variety. This observation is consistent with recent modelling of distributions of incubation periods (Ottino-Loffler et al., 2017), but it contradicts the established view in the literature on $Z$. tritici according to which lesions

445 appear after an asymptomatic period of approximately two weeks (Kema et al., 1996; Steinberg, 2015). Shaw (1990) used pycnidiospores from the natural field population of $Z$. tritici to inoculate wheat plants in the greenhouse and found that lesions were appearing continuously during the period of up to 25-30 days. However, in the experiments of Shaw

450 (1990), the asynchronicity in lesion appearance could result from both the variation in the latent period between different pathogen strains and the "developmental" asynchronicity within a single pathogen genotype that we observed here. Recently Fones et al. (2017) have shown that there is a large variation in the timing of penetration events of spores originating

455 from the same inoculation event. They observed spores on leaf surfaces 
until $10 \mathrm{DPI}$ and found that the fraction of successfully penetrating individuals was continuously increasing. This variation in the duration of the epiphytic growth offers a possible explanation for large differences in the times of appearance of individual lesions that we observed. At higher 460 inoculum concentrations, lesions started to appear earlier possibly due to a higher total number of infections that led to an increase in the number of rare "fast" infections. Consequently, the large variation in the duration of the asymptomatic period that we observed at low inoculum concentrations is likely hidden in experiments that use high inoculum

465 concentrations [e.g., $10^{6}$ spores $/ \mathrm{ml}$ is typically used in greenhouse trials, for example in (Stewart \& McDonald, 2014)]. In this case, leaves become fully necrotic only a few days after the appearance of first lesions and the appearance of new lesions is no longer visible.

In treatments with low densities of only 1-5 spores $/ \mathrm{cm}^{2}$, it is highly

470 plausible that individual lesions were caused by single spores. This is consistent with experimental findings of Shaw, (1990), in which the inoculations were conducted with the population of natural field strains of $Z$. tritici. Fones et al., (2015) confirmed this finding using the reference strain of $Z$. tritici IPO323. This may not be the case at higher spore

475 densities when the area covered by a typical lesion contains many viable spores and the spores may cooperate to cause infection and form a lesion. Indeed, $Z$. tritici is able to undergo anastomosis between germinating spores when they are deposited on the leaf close to each other (Mehrabi et al., 2009). Spores may also interact in more subtle ways, as was

480 observed in other foliar plant pathogens (Jeffries, 1995, Jesus Junior et al., 2014). For example, when two spores penetrate the leaf surface at nearby locations, the probability of lesion formation may become higher (cooperation) or lower (antagonism) than twice the probability to form a lesion by an individual spore that is far away from other spores. Also,

485 when one spore causes a lesion, the probability of other spores in the vicinity to cause a lesion may increase (cooperation) or decrease 
(antagonism). Such interactions should lead to departures from a linear dependency between the density of spores and the density of lesions of leaves. According to our measurements, this dependency is linear within a 490 wide range of spore densities, from low densities of $1-5 \mathrm{spores} / \mathrm{cm}^{2}$ to intermediate densities of $10-80$ spores $/ \mathrm{cm}^{2}$. This indicates that in this range of spore densities, lesions are caused by single spores with no evidence of interaction between spores within the limits of accuracy of our measurements.

495 Interaction between spores may still occur at higher spore densities, but in our experiment, its effect would not be visible because of the saturation of the leaf surface with lesions.

Some evidence for a possible cooperation between spores belonging to the same strain in causing lesions was provided by Haueisen et al. (2017).

500 After inoculating wheat leaves with a suspension containing a high concentration of $Z$. tritici spores $\left(10^{8}\right.$ spores $\left./ \mathrm{ml}\right)$, they found cases when several hyphae entered a single stoma. Additionally, noticed that the spatial distribution of stomatal penetrations appeared to be clustered, rather than uniform. Some indication for an antagonistic interaction

505 between different $Z$. tritici strains in pycnidia formation was reported by Schuster et al. (2015): each pycnidium was produced by a single strain when two strains were inoculated together even though hyphae of the two strains were found growing next to each other.

Our estimates of infection efficiency were close to $4 \%$ in both biological 510 replicates. However, statistical tests revealed that the difference in the estimates between the two replicates was significant. Several factors may be responsible for the difference. The experiment was conducted under controlled greenhouse conditions but changes in the external weather may still have affected the outcomes. While the first experiment was

515 performed in early January, the second experiment was performed in the 
beginning of March. Hence, both the amount of external light and the external temperature differed between the experiments. In addition, during the second experiment, we observed several times water droplets on infected leaves and aluminium plates. This may have caused

520 microclimatic differences between the two replicates and such differences are known to affect infection success of $Z$. tritici (Shaw, 1990). The fungal inoculum was grown from the same batch and prepared with the same protocol in both replicates, but the fungus is capable of rapidly accumulating genetic changes through mitotic events (Möller et al., 2018)

525 and this may have contributed to the difference in the estimates of infection efficiency.

Infection efficiency of $Z$. tritici blastospores of strain IPO323 was estimated to be around $50 \%$ on wheat cultivar Galaxie by Fones et al. (2015), which is much higher than the estimate of around $4 \%$ that we

530 report here. Many factors may have contributed to this difference. Aggressiveness of the two pathogen strains may be different as well as the degree of resistance of the two wheat cultivars. Greenhouse conditions in the experiment by Fones et al. (2015) differed from our experiment in terms of light and temperature. Also, the age of infected

535 seedlings was only 10 days compared to 16 days in our experiment. Another major factor could be the difference in the inoculation methods. They pipetted droplets of the spore suspension on the leaf surface and spread them with a gloved finger, while we sprayed the spore suspension creating a fog consisting of microscopic water droplets. When spread with

540 a finger, spores were likely to be placed close to the leaf surface. In contrast, a substantial fraction of water droplets in the fog may have remained on trichomes (leaf hairs), which could diminish their penetration success. Our estimate for infection efficiency of $Z$. tritici, about $4 \%$, lies within the lower range of what was previously reported in the literature for

545 other fungal pathogens. Among 22 infection efficiency estimates in different species discussed by Sache $\&$ de Vallavieille-Pope (1995), eight 
species had the values within the range of $0-5 \%$, five species ranged within $6-15 \%$, five species ranged within $16-25 \%$ and four species ranged within 26-50\%. Notably, Sache \& de Vallavieille-Pope (1995) used the

550 highest value for each species they found in the literature. More recent research on measuring the infection efficiency of fungal plant pathogens is scarce, but Li et al. (2010) estimated the infection efficiency of soybean rust (Phakopsora pachyrhizi) to be within the range of $0.5-10 \%$.

We infected a single wheat variety with blastospores of a single strain of

$555 Z$. tritici to provide a proof of principle for the reliable measurement of the infection efficiency. Using blastospores is a standard method to conduct greenhouse trials with this pathogen, but the role of blastospores in the pathogen's life cycle remains unknown. However, our method could also be used to measure the infection efficiency of both pycnidiospores and

560 ascospores, which are known to drive the epidemics in the field. Infection efficiency may be different in other pathogen strains or when infecting other wheat varieties. Haueisen et al. (2017) observed that three strains of $Z$. tritici differed in terms of the time between the inoculation and the first stomatal penetration and in terms of the degree of epiphyllous

565 proliferation. We also expect to observe specialization of pathogen strains to certain wheat varieties, because the pathogen population is extremely diverse (Linde et al., 2002) and is known to adapt rapidly to different host environments (Poppe et al., 2015). These factors may lead to different infection efficiencies in different $Z$. tritici strains. Therefore, we do not

570 expect that our estimate of infection efficiency in this particular pathogen strain-host cultivar combination would be representative of the natural pathogen population. However, using this method, a number of pathogen strains can be tested on a number of wheat varieties. Such a comprehensive characterization of infection efficiency would improve the

575 predictive power of mathematical models that describe epidemic development and pathogen evolution and in this way contribute to improving control strategies. If a sufficient degree of heritable variation in 
terms of infection efficiency is found in the pathogen population, the genetic basis of this trait could be revealed, for example by conducting a

580 genome-wide association study. Similarly, from the perspective of the host, our method opens opportunities for determining the genetic basis of the component of quantitative resistance that suppresses infection efficiency. This knowledge has the potential to improve and accelerate breeding for quantitative resistance against STB.

\section{Acknowledgements}

P. Karisto and A. Mikaberidze gratefully acknowledge financial support from the Swiss National Science Foundation through the Ambizione grant PZOOP3161453. The authors would like to thank Bruce A. McDonald and the members of the Plant Pathology group at the ETH Zürich for fruitful 590 discussions. 


\section{References}

Brown JF, Kochman JK, 1973. A spore settling tower for uniform inoculation 595 of leaves with rust urediniospores. Australasian Plant Pathology 2, 26-7.

Chakraborty S, Ratcliff D, McKay FJ, 1990. Anthracnose of Stylosanthes scabra: Effect of leaf surface wetness on disease severity. Plant Dis. 74: 379-384.

600 Cowger C, Hoffer ME, Mundt CC, 2000. Specific adaptation by Mycosphaerella graminicola to a resistant wheat cultivar. Plant Pathology 49, 445-51.

Cowger C, Mundt CC, 2002. Aggressiveness of Mycosphaerella

605 graminicola Isolates from Susceptible and Partially Resistant Wheat Cultivars. Phytopathology 92, 624-30.

Fones HN, Gurr SJ, 2015. The impact of Septoria tritici Blotch disease on wheat: an EU perspective. Fungal Genetics and Biology 79, 3-7.

Fones HN, Steinberg G, Gurr SJ, 2015. Measurement of virulence in Zymoseptoria tritici through low inoculum-density assays. Fungal Genetics and Biology 79, 89-93.

615 Fones HN, Eyles CJ, Kay W, Cowper J, Gurr SJ, 2017. A role for random, humidity-dependent epiphytic growth prior to invasion of wheat by Zymoseptoria tritici. Fungal Genetics and Biology 106, 51-60.

Fraaije B, Cools HJ, Fountaine J et al., 2005. Role of Ascospores in Further

620 Spread of Qol-Resistant Cytochrome $b$ Alleles (G143A) in Field Populations of Mycosphaerella graminicola. Phytopathology 95, 933-41.

Haueisen J, Moeller M, Eschenbrenner CJ et al., 2017. Extremely flexible infection programs in a fungal plant pathogen. bioRxiv 229997 doi:

$62510.1101 / 229997$.

Jeffries P, 1995. Biology and ecology of mycoparasitism. Canadian journal of botany 73, 1284-90.

630 Jesus Junior WC, Paula Júnior TJ, Lehner MS, Hau B, 2014. Interactions between foliar diseases: concepts and epidemiological approaches. Tropical Plant Pathology 39, 1-18. 
Jørgensen LN, Hovmøller MS, Hansen JG et al., 2014. IPM Strategies and

635 Their Dilemmas Including an Introduction to www.eurowheat.org. Journal of Integrative Agriculture 13, 265-81.

Karisto P, Hund A, Yu K et al., 2018. Ranking Quantitative Resistance to Septoria Tritici Blotch in Elite Wheat Cultivars Using Automated Image

640 Analysis. Phytopathology 108, 568-81.

Kema GH, Yu D, Rijkenberg FH, Shaw MW, Baayen RP, 1996. Histology of the pathogenesis of Mycosphaerella graminicola in wheat. Phytopathology 86, 777-86.

645

Keon J, Antoniw J, Carzaniga R et al., 2007. Transcriptional Adaptation of Mycosphaerella graminicola to Programmed Cell Death (PCD) of Its Susceptible Wheat Host. MPMI 20, 178-93.

650 King R, Urban M, Lauder RP et al., 2017. A conserved fungal glycosyltransferase facilitates pathogenesis of plants by enabling hyphal growth on solid surfaces. PLOS Pathogens 13, e1006672.

Lee W-S, Rudd JJ, Hammond-Kosack KE, Kanuyka K, 2014. Mycosphaerella 655 graminicola LysM Effector-Mediated Stealth Pathogenesis Subverts Recognition Through Both CERK1 and CEBiP Homologues in Wheat. MPMI 27, 236-43.

Levy $Y, 1989$. Analysis of epidemics of northern leaf blight on sweet corn 660 in Israel. Phytopathology 79, 1243-5.

Li X, Mo J, Guo T, Yang X, 2010. Effects of light on urediniospore germination, appressorium formation and infection efficiency of Phakopsora pachyrhizi, causal agent of soybean rust. Canadian Journal of 665 Plant Pathology 32, 153-61.

Linde C, Zhan J, McDonald BA, 2002. Population structure of Mycosphaerella graminicola: From lesions to continents. Phytopathology 92, 946-55.

Mehrabi R, M'Barek SB, van der Lee TA, Waalwijk C, de Wit PJ, Kema GH, 2009. $G \alpha$ and $G \beta$ proteins regulate the cyclic AMP pathway that is required for development and pathogenicity of the phytopathogen Mycosphaerella graminicola. Eukaryotic cell 8, 1001-13.

Mehta Y, Zadoks J, 1970. Uredospore production and sporulation period of Puccinia recondita f. sp. triticina on primary leaves of wheat. Netherlands journal of plant pathology 76, 267-76. 
680 Melching J, Dowler W, Koogle D, Royer M, 1988. Effect of plant and leaf age on susceptibility of soybean to soybean rust. Canadian Journal of Plant Pathology 10, 30-5.

Möller M, Habig M, Freitag M, and Stukenbrock EH, 2018. Extraordinary 685 genome instability and widespread chromosome rearrangements during vegetative growth. bioRxiv doi: 10.1101/304915.

Noblin X, Yang S, Dumais J, 2009. Surface tension propulsion of fungal spores. Journal of Experimental Biology 212, 2835-2843.

Orton ES, Deller S, Brown JK, 2011. Mycosphaerella graminicola: from genomics to disease control. Molecular plant pathology 12, 413-24.

Ottino-Loffler B, Scott JG, Strogatz SH, 2017. Evolutionary dynamics of 695 incubation periods. eLife 6, e30212.

Palma-Guerrero J, Torriani SFF, Zala M et al., 2016. Comparative transcriptomic analyses of Zymoseptoria tritici strains show complex lifestyle transitions and intraspecific variability in transcription profiles

Parlevliet JE, 1979. Components of resistance that reduce the rate of epidemic development. Annual Review of Phytopathology 17, 203-222.

Poppe S, Dorsheimer L, Happel P, Stukenbrock EH, 2015. Rapidly evolving 705 genes are key players in host specialization and virulence of the fungal wheat pathogen Zymoseptoria tritici (Mycosphaerella graminicola). PLoS pathogens 11, e1005055.

R Core Team, 2017. R: A language and environment for statistical

710 computing. R Foundation for Statistical Computing, Vienna, Austria. [https://www.R-project.org].

Risser $\mathrm{P}, 2010$. Mapping of quantitative-trait loci (QTL) for adult-plant resistance to Septoria tritici in five wheat populations (Triticum aestivum

715 L.). Stuttgart, Germany: University of Hohenheim, PhD thesis. [http://opus.ub.uni-hohenheim.de/volltexte/2010/517/].

Sache I, 1997. Effect of density and age of lesions on sporulation capacity and infection efficiency in wheat leaf rust (Puccinia recondita f. sp. tritici). Plant Pathology 46, 581-9.

Sache I, de Vallavieille-Pope C, 1995. Classification of airborne plant pathogens based on sporulation and infection characteristics. Canadian Journal of Botany 73, 1186-95. 
Schuster M, Kilaru S, Guo M, Sommerauer M, Lin C, Steinberg G, 2015. Red fluorescent proteins for imaging Zymoseptoria tritici during invasion of wheat. Fungal Genetics and Biology 79, 132-40.

730 Septoria tritici blotch network, 2017. Zymoseptoria tritici isolate collections made by different groups. [http://www.septoria-triticiblotch.net/isolate-collections.html]. Accessed 9 April 2018.

Shaw MW, 1990. Effects of temperature, leaf wetness and cultivar on the 735 latent period of Mycosphaerella graminicola on winter wheat. Plant Pathology 39, 255-68.

Shine Jr J, Jarriel W, 1990. Study of the development of sugarcane rust Puccinia melanocephala, uredini by artificial inoculation of highly

740 susceptible sugarcane clones. Journal-American Society of Sugar Cane Technologists 10, 73-8.

Statler GD, McVey MA, 1987. Partial resistance to Uromyces appendiculatus in dry edible beans. Phytopathology 77, 1101-3.

St.Clair DA, 2010. Quantitative disease resistance and quantitative resistance loci in breeding. Annual Review of Phytopathology 48, 247-68.

Steinberg G, 2015. Cell biology of Zymoseptoria tritici: Pathogen cell

750 organization and wheat infection. Fungal Genetics and Biology 79, 17-23.

Stewart EL, McDonald BA, 2014. Measuring quantitative virulence in the wheat pathogen Zymoseptoria tritici using high throughput automated image analysis. Phytopathology 104, 985-92.

Suffert F, Sache I, Lannou C, 2011. Early stages of septoria tritici blotch epidemics of winter wheat: build-up, overseasoning, and release of primary inoculum. Plant Pathology 60, 166-77.

760 Valsecchi I, Sarikaya-Bayram Ö, Wong Sak Hoi J et al., 2017. MybA, a transcription factor involved in conidiation and conidial viability of the human pathogen Aspergillus fumigatus. Molecular Microbiology 105, 880900.

765 Zar JH, 2010. Biostatistical analysis ( $5^{\text {th }}$ edition). Upper Saddle River, NJ, USA: Pearson Prentice Hall. Chapter 18.

Zhan J, Kema G, Waalwijk C, McDonald BA, 2002. Distribution of mating type alleles in the wheat pathogen Mycosphaerella graminicola over

770 spatial scales from lesions to continents. Fungal Genet. Biol. 36, 128-136. 
Zhan J, Stefanato F, McDonald BA, 2006. Selection for increased cyproconazole tolerance in Mycosphaerella graminicola through local adaptation and in response to host. Molecular Plant Pathology 7, 259-68.

775 
Supporting information legends

Appendix S1 Detailed description of the outcomes of the spraying method used for inoculation.

Figure S1 Representative reference membranes showing colonies of $Z$. 780 tritici.

Figure S2 Microscopic images of sprayed spores.

Figure S3 Dependency of the colony density on the spore concentration in the inoculum suspension. 


\section{Figure legends}

Figure 1 (a) Experimental setup. Second leaves were attached to metal plates by elastic threads. Wooden sticks were used to stabilize individual pots and to support the plastic bags during the high humidity period after the inoculation. Reference membranes were placed on both sides of each

790 leaf set during the inoculation. (b,c,d) Representative leaf sets at 14,17 and 23 days post inoculation from left to right corresponding to the concentration of spores of $5 \times 10^{2}$ spores $/ \mathrm{ml}$ (b), $10^{4}$ spores $/ \mathrm{ml}$ (c), and $5 \times 10^{4}$ spores $/ \mathrm{ml}(\mathrm{d})$. Lesions are marked with coloured dots that indicate the day and the position of their first appearance.

795 Figure 2 Appearance of lesions. The number of lesions that appeared since the previous observation as a function of time in the first experiment (a) and in the second experiment (b). Total number of lesions in the first experiment (c) and in the second experiment (d). The values were divided by the total leaf area separately in each treatment. Note the logarithmic 800 scale on the $y$-axis; values are added by 0.01 to show zeros. Different colors/symbols represent treatments with different spore concentrations in the inoculum suspension: purple squares $\left(5 \times 10^{2}\right.$ spores $\left./ \mathrm{ml}\right)$; blue diamonds $\left(10^{3}\right.$ spores $\left./ \mathrm{ml}\right)$; yellow filled triangles $\left(5 \times 10^{3}\right.$ spores $\left./ \mathrm{ml}\right)$; red diamonds $\left(10^{4}\right.$ spores $\left./ \mathrm{ml}\right)$; green open triangles $\left(5 \times 10^{4}\right.$ spores $\left./ \mathrm{ml}\right)$; cyan 805 stars $\left(10^{5}\right.$ spores $\left./ \mathrm{ml}\right)$.

Figure $\mathbf{3}$ Infection efficiency of $Z$. tritici spores. (a) The lesion density is plotted against the spore density; (b) magnified view of the low-density part of panel (a). Data and curves are shown in grey for the first experiment and in black for the second experiment. Different symbols

810 correspond to treatments with different concentrations of spores in the inoculum, same as in Fig. 2. Dashed lines show the nonlinear function 
$y=a x /(1+b x)$ fitted to full data of each experiment. Solid lines show the linear function $\mathrm{y}=\mathrm{ax}$ fitted to data below $100 \mathrm{spores} / \mathrm{cm}^{2}$. 